\title{
Systems consolidation and hippocampus: two views
}

\author{
Lynn Nadel • Gordon Winocur • Lee Ryan • \\ Morris Moscovitch
}

Received: 27 September 2006 / Accepted: 17 January 2007 /Published online: 6 March 2007

(C) Springer Science + Business Media, LLC 2007

\begin{abstract}
Two approaches to systems-level memory consolidation are contrasted. The standard model and multiple trace theory are spelled out, their implications are outlined, and their fit to the data from a number of approaches is evaluated. We conclude that the data from neuroimaging studies strongly support multiple trace theory, that data from neuropsychological studies favor but does not conclusively support multiple trace theory, while evidence from a new approach, the study of prospective memory, also supporting multiple trace theory, offers a promising new way to distinguish between these two theories. Work with animals is largely consistent with this conclusion. We suggest that the hippocampal and neocortical systems are critical for different forms of memory, and that the shift of memory from dependence on hippocampus to dependence on neocortex during consolidation is a reflection of the fact that memory often is transformed with time, becoming more generic in nature. Insofar as detailed episodic recollections are retained, the data show that they are
\end{abstract}

\footnotetext{
L. Nadel $(\bowtie) \cdot$ L. Ryan

Department of Psychology, University of Arizona,

Tucson, AZ, USA

e-mail: nadel@u.arizona.edu

G. Winocur

Rotman Research Institute, Baycrest Toronto,

Toronto, ON, Canada

G. Winocur $\cdot$ M. Moscovitch

Department of Psychology, University of Toronto,

Toronto, Ontario, Canada

G. Winocur

Department of Psychology, Trent University,

Peterborough, ON, Canada
}

dependent on the hippocampal system, much as multiple trace theory postulated.

Keywords systems consolidation · hippocampus . multiple trace theory $\cdot$ context

The idea that memories are "consolidated" at the systems level emerged about 60 years ago from two key findings: Russell and Nathan [73] showed that the retrograde amnesia (RA) resulting from brain trauma can last days or even months, and Scoville and Milner [77] showed that resection of the hippocampus apparently caused both anterograde amnesia and RA, the latter lasting as long as several years. Because it was thought that the short-term (STM) and long-term memory (LTM) capacities of the patient H.M. were intact, it was concluded that parts of the medial temporal lobe (MTL) played some critical, although unspecified, role in transferring memories from STM to LTM. Interest focused quite soon on the hippocampus within the MTL (see [65]).

For some time this notion of transfer was taken literally; it was assumed that a given memory was initially "stored" in hippocampal circuits, and then somehow transferred or reestablished in extrahippocampal (likely neocortical) memory sites. Perhaps the first statement of this position was contained in Marr's [44] model of the hippocampal system. The more general idea that systems consolidation (or prolonged consolidation, compared to synaptic [Dudai 2004] or rapid consolidation [Moscovitch 2000] involved long-term interactions between hippocampal and extrahippocampal circuits was expressed in an article by Squire et al. [79]. The "standard model of memory consolidation" (henceforth SMC) emerged from these writings. It is important to note at the outset that this model was originally intended to apply to explicit forms of memory, 
including both episodic and semantic memory, ${ }^{1}$ although these terms were not in use in the 1950s and 1960s (see [60] for a recent discussion of the emergence of ideas about multiple memory systems). The fact that early discussion of systems consolidation concerned only one type of memory is important in understanding the theories that emerged and their limitations. More recently it has become clear that systems-level consolidation, understood as interactions between multiple brain systems influencing LTM storage, might be occurring also for implicit forms of memory, but in this case the relevant structures are other than the hippocampus and its neighbors in the MTL. ${ }^{2}$ Our focus here will be on explicit memories and the standard model offered to explain how these kinds of memories are consolidated, because this is really what the historical debate is all about. It will be interesting in the future to ask whether the principles that apply to systems consolidation in explicit memory are also relevant for implicit memory.

The standard model supposes that the hippocampus plays a time-limited role in memory storage, either by first storing and then transferring a memory, or by acting as an index (cf. [83] for an early formulation of this indexing idea) that binds extrahippocampal storage sites during a period when direct communication among these sites is inadequate to permit memory retrieval. As a function of replay, either during rehearsal or offline, these extrahippocampal sites acquire the ability to interact such that the hippocampus is no longer needed to retrieve the memory. Initially a supporting argument held that there is limited capacity within hippocampal circuits, creating a need to recycle hippocampal neurons back into a pool of available

\footnotetext{
${ }^{1}$ There is considerable disagreement about which terms to use in referring to various kinds of memory. Whereas episodic and semantic memory are relatively well defined, the umbrella terms embracing both of these, explicit or declarative memory, are each fraught with difficulty. The former, with its partner implicit memory, was introduced not as the name for a memory system, but rather as a description of the methods used to assess it. The latter, with its original partner procedural memory, was introduced as a name for a memory system, but it has proven virtually impossible to define in a noncircular way. We prefer the theory-neutral nature of the terms "explicit" and "implicit," while acknowledging that explicit memory is not a "system."

${ }^{2}$ The terminology for MTL structures is not consistent in the literature. Here, the hippocampus proper refers to the cornu ammonis fields and dentate gyrus. The hippocampal formation includes the hippocampus proper and the subiculum, whereas the hippocampal complex includes the hippocampal formation plus entorhinal and perirhinal cortex, and the parahippocampal cortex. Both SMC and MTT discuss consolidation with respect to the hippocampus proper. Whereas proponents of both theories recognize the importance of other MTL structures in memory encoding and retrieval, neither theory makes strong claims regarding the specific roles these structures may play, or how they interact with one another.
}

elements for new memory formation (e.g., [3]). When extrahippocampal sites can interact to retrieve memories, the hippocampus is no longer needed and systems-level memory consolidation is said to be complete. Before this time normal memory retrieval requires hippocampal involvement. Within this model little was said about the time course of changes in the strength of memory representations in hippocampal and extrahippocampal circuits. Is it because the former is weakening even as the latter is strengthening? If so, how could they still support retrieval? If not, are hippocampal traces suddenly erased just when extrahippocampal traces reached coherency? Or does the hippocampus continue to contribute as it did before consolidation, but in a way that is complementary rather than necessary? Questions of this kind were rarely, if ever, asked.

Notwithstanding the lack of the kind of details just noted, SMC gained rapid acceptance in the field (cf. [78]), entering the textbooks as a fact about memory and the brain. Questions about this approach came from researchers working both with humans (e.g., [88-90]; Kinsbourne and Wood 1975) and with animal models (e.g., [38]), but their concerns were not considered sufficient to challenge the main ideas expressed in the standard view. One frequently sees uncritical acceptance of SMC in both the scientific and nonscientific literature - the hippocampus, in this view, is only important for memory for a brief interlude.

\section{A challenge to the standard theory}

About 10 years ago we began to question SMC seriously [51, 61, 62]. Based on a comprehensive review of the existing literature we concluded that SMC suffered from quite serious problems. Some of these were empirical, whereas others were conceptual, and several were severe enough to cast doubt on SMC.

\section{Problems with SMC}

The problems with SMS are as follows:

1. The apparent extent of RA, hence the length of the consolidation period, varies with both the memory task being tested and the method used to produce amnesia, e.g., lesions, electroconvulsive shock, immersion in cold water, or the injection of protein synthesis inhibitors. To complicate matters further, the animal literature measures consolidation in hours, days, or weeks at most, whereas the human neuropsychological literature measures consolidation in years, if not decades.

2. Insofar as one imagines a transfer of memory from hippocampal to extrahippocampal sites, no empirically 
supported mechanism to accomplish this has been offered. If one imagines that memory is always stored in extrahippocampal sites, but requires hippocampal involvement early on for coherent retrieval, then no mechanism is proposed by virtue of which coherent retrieval without hippocampal involvement becomes possible. Do connections that already exist become stronger? Do new connections grow among the neuronal populations involved? Is there any evidence for either of these possibilities? (but see Murre et al. 2002 for a discussion of recent attempts to address these issues).

3. The SMC, treating episodic and semantic memory as equivalent, offers no account of how, over time, episodic memories appear to become more semantic in nature. That is, we know that over time episodic detail is lost whereas "gist" is preserved, but SMC offers no way of understanding how and why this happens. The model proposed by McClelland et al. [48] does address this question, but in the process it ignores the continuing presence of episodic memory.

Given these and other weaknesses of the standard theory, Nadel and Moscovitch [61] generated a new model called the multiple trace theory (henceforth MTT; see Estes 1955 and Hintzman 1986 for mention of multiple traces in cognitive models of anterograde memory), which sought to account for the facts of systems-level consolidation in a novel way. This model built on the "cognitive map theory" of hippocampal function [64] and the component process model $[49,50]$ but went beyond them in a number of ways (cf. [55] for a comprehensive discussion of the relations among these models).

MTT made a range of predictions, most prominently the idea that retrieval of remote memories would engage the hippocampal complex, in contrast to what SMC predicted. In the remainder of this paper we will review the current literature, both in studies with humans and other animals, with an eye toward resolving this debate (see Moscovitch et al. [53-55] for extensive treatment of many of these issues).

We start with more precise statements of both SMC and MTT, focusing on the central predictions of each position. We then review the literature. We review studies in humans using both neuroimaging and neuropsychological methods; each approach allows one to ask different questions. We follow with a short discussion of studies in experimental

\footnotetext{
${ }^{3}$ This prediction reflects the initial formulation of SMC before the advent of neuroimaging methods. Such methods make it clear that hippocampus is activated when remote memories are retrieved. Updated versions of SMC argue that such activation reflects encoding of new memories rather than retrieval of remote ones (see below).
}

animals (mostly rodents), which have tried to unravel some of the thornier questions that often cannot be asked in studies with humans. We conclude that although some issues remain to be resolved, the weight of the evidence at present strongly favors MTT.

\section{The two positions explained}

SMC and its predictions

According to SMC, in its current incarnation, all explicit memories (e.g., both episodic and semantic) are always stored in extrahippocampal circuits. The hippocampal contribution is in the form of an "index," which serves to bind together the disparate neocortical sites representing the various parts of an episode, or the semantic structure abstracted from related episodes. This position seems to make a number of critical predictions that contrast it with MTT:

1. All the "information" inherent in an explicit memory is to be found in neocortical circuits, not in hippocampal circuits. Thus, a memory, independent of the hippocampus, contains the same information that it would contain were the hippocampal system available, either before or after the completion of consolidation (cf. p. 424 in [48]). The implication is that remote episode memories retrieved by individuals with amnesia must be as fully detailed as remote episode memories retrieved by individuals with intact brains. Note that this need not imply that remote memories contain as much detail as more recent memories, just that the absence of the hippocampus cannot influence the qualitative nature of a memory retrieved after it is consolidated (cf. p. 141 in [5]), assuming that such consolidation happened while the hippocampus was intact.

2. Given that the hippocampus is not involved in retrieval of remote memories, such retrieval should not be accompanied by activation in the hippocampus. ${ }^{3}$

3. Both forms of explicit memory, episodic and semantic, are treated the same with regard to systems consolidation. Both start out dependent upon the hippocampus, and both end up independent of it.

\section{MTT and its predictions}

According to MTT, memories are stored jointly in hippocampal and extrahippocampal circuits, and rather different things happen to episodic and semantic memory. This leads to a somewhat different set of predictions: 
1. The information inherent in an episodic memory is contained in both hippocampal and extrahippocampal circuits, such that remote episode memories retrieved by individuals with amnesia should not be qualitatively the same as remote episode memories retrieved by individuals with intact brains.

2. Given that the hippocampus is involved in retrieval of certain aspects of remote episode memory, such retrieval should be accompanied by activation in the hippocampus.

3. The two forms of explicit memory, episodic and semantic, are not treated the same with regard to systems consolidation. According to MTT, episodic memories always remain dependent upon hippocampal circuits, at least for their most detailed expression. Semantic memories, on the other hand, can be independent of hippocampal circuitry in just the way standard theory suggests. That is, semantic information is always represented in extrahippocampal circuits, and the process of consolidation acts to strengthen these circuits and to integrate newly acquired semantic information into existing stores.

\section{Contrasting predictions}

Given these distinctions, it is clear that three areas of empirical investigation and three seemingly simple questions are central to distinguishing between SMC and MTT:

1. Are remote memories retrieved by amnesics as fully detailed as remote memories retrieved by intact individuals (SMC) or are there qualitative differences (MTT)?

2. Is the hippocampus activated by retrieval of remote episodic memories (MTT) or not (SMC)?

3. Do episodic and semantic memories suffer the same fate during consolidation and in amnesia (SMC) or are they affected differently (MTT)?

Although these questions appear to be simple ones, of course they turn out to be much more complicated once one tries to answer them definitively. To start, the first question raises complex issues about the methods used to elicit remote memory retrievals. The second question can only be addressed in neuroimaging contexts, and runs into the problem of separating retrieval from encoding. The third question itself begs the issue of the relation between episodic and semantic memory. In the literature review that follows, we highlight these problems while trying to reach a conclusion about the merits of the two theoretical positions.

\section{The nature of remote memory in human amnesics}

Episodic memory

The initial formulation of SMC and views of systems consolidation in general had consolidation limited to weeks in rodents and monkeys and to about 3-10 years, at the outside, in humans. It is now clear, contrary to these early claims, that large MTL lesions produce a RA that extends for decades, consistent with the initial formulation of MTT, that the extent and severity of RA is determined by the size of MTL lesions (for different evidence regarding this prediction, see Kopelman et al. 2004; Gilboa et al. 2005). Having abandoned their earlier position and conceded that $\mathrm{RA}$ is extensive, proponents of SMC now focused their major point of contention on whether episodic, autobiographical memory from the most remote time periods, usually childhood and early adulthood, is preserved or impaired when damage is limited to the MTL. Another issue is whether damage limited to the hippocampal formation can produce a temporally extensive RA at all. Proponents of MTT and SMC have offered functional and anatomical accounts for the current discrepancy among studies, respectively.

According to the functional account favored by MTT, hippocampal involvement in episodic memory is defined by the recollective quality and detail of the remembered event. Because most autobiographical memories lose their vividness and detail as they age [36], memories of amnesic and normal people may appear equally impoverished at remote periods if memory is queried insufficiently. The Autobiographical Memory Interview (AMI) [31], a standard test of remote episodic and semantic personal memory used in most studies, may reveal differences between controls and amnesics in some cases. In other cases, however, special interviewing and scoring procedures may be needed. For example, in the patient H.M., such tests have revealed extensive memory loss dating to early life [81], although on less sensitive tests his RA appeared more limited, leading to the conclusion that his remote memories were relatively normal [11, 77].

Although Bayley et al. [5, 6], using more sensitive tests, still reported no difference between amnesic people with large MTL lesions and controls at the most remote time period, these data should be interpreted with caution. The number of details recalled by their controls was far less than that reported by controls in other studies [36], nor was there any evidence of the typical loss of memory with aging in their control subjects, a finding inconsistent with most of the work in this area. Differences in testing procedure and scoring may account for some of these discrepancies. What is more worrying, however, is this group's report that patients with lesions confined to the MTL had ungraded 
deficits in semantic memory lasting at least 40 years in one study [68]. It is highly unusual for RA to be far more severe for semantic than episodic memory in such cases, suggesting that the method used by Bayley et al. to test episodic memory may not have been sensitive enough.

This point is brought out most clearly in their most recent study. Despite evidence regarding the insensitivity of the AMI, Bayley et al. (2006) used it to assess remote autobiographical memory. Not surprisingly, memory was hardly affected even for events occurring in early adulthood in people with extensive MTL damage, who also had substantial damage to the insula and inferior temporal cortex. Interestingly, however, these very same patients had an extensive RA for public (news) events, which extended to over 50 years in one case. One reason is that it was much more difficult to obtain a high score on the news events tests, which included quite difficult items (e.g., What tire manufacturer recalled thousands of tires [answer: Firestone]) compared to the test for autobiographical memory, which depended on a laxer and more subjective scoring method. Thus, whereas controls performed at or near ceiling on the AMI, receiving scores close to the maximum of $9 / 9$, they scored at only $60 \%$ correct for recent news events, and dropped to $40 \%$ correct for news events that occurred 10 or more years earlier. These findings underscore the need to use a comparable metric for testing both types of memory, as Steinvorth et al. [81] did in their study.

The anatomical account favored by SMC attributes the difference between extensive and temporally limited RA to lesion size and location. Drawing on carefully documented neuroanatomical analysis of a series of amnesic patients, Bayley et al. conclude that RA encompassing the earliest periods of life is found only in patients whose damage extends beyond the MTL to regions of the neocortex, a finding supported, in part, by Bright et al. (2006). In their view, damage confined to the MTL spares memories for the most remote periods, although in the study of Bright et al, there is a suggestion that memory is impaired at the most remote period even in people with lesions confined to the hippocampus proper. The deficit fails to reach significance probably because of the low power of the test caused by variability in performance and the low number of subjects tested. Other cases, however, show RA across the life span even when the lesions are confined to the hippocampal formation (see Table 1 in [55]), and more specifically just to the fornix [67], which contains the major projections of the hippocampus. At least one of those cases, V.C., is as well documented neuroanatomically as the cases in the series of Bayley et al. [8] and, except for memory loss, is at least as cognitively intact. Moreover, the cases of Bayley et al. with the most extensive damage to MTL also have considerable damage to the insula and inferior temporal cortex, indicating that it is not likely to be the extent of extrahippocampal damage that accounts for the differences in RA observed among the different cases, but the way in which memory was evaluated.

Thus, neither the functional nor anatomical accounts seem adequate at this point because there are reports of ostensibly vivid, remote memories being spared (Bayley et al. [5, 6]) and cases with damage limited to the hippocampus proper, MTL, or its projections, who show impaired memory even at the most remote periods [8] (for more discussion, see [53-55]). For many of the same reasons, other issues are also unresolved, among them, the extent and nature of focal RA, of transient global amnesia, of the correlation between lesion size and extent of deficit ([29, 30, 32]; Gilboa et al. 2005), the unique contribution, if any, of the different regions of the MTL, and of memory loss after different types of dementia $[25,56,59]$. We believe that on balance the neuropsychological evidence concerning the status of remote memory in amnesia favors MTT, but we are aware that some of the studies are inconclusive, and others are open to different interpretations.

Some of the methodological problems associated with studying the role of the hippocampus in remote memory retrieval may be side-stepped by taking advantage of the possibility that the same brain system enabling backward mental time travel, e.g., memory retrieval, might also be critically engaged in imagining events that never happened, or that might happen in the future. This idea, brought to prominence by Tulving [86] and discussed recently by Schacter and Addis [76], has recently been tested in several studies. Ryan et al. [74] showed that the hippocampus is activated by such imaginings (see below). Given this, one wonders whether amnesic patients can imagine fictional events, either in the past or in the future. Rosenbaum et al. (2003) showed that patient K.C. was markedly impaired at imagining fictional events. Hassabis et al. [23] tested a group of amnesic patients more extensively on their ability to imagine specific future scenarios. All but one of the five patients showed profound impairment at this task. ${ }^{4}$ What is more, the nature of the defect was quite revealing. Patients were incapable of generating a holistic representation of any imagined environment within which experiences could achieve coherence. As a consequence, they could only generate fragments that never achieved the status of an event. The authors conclude that the hippocampus "may make a critical contribution to the creation of new experiences by providing the spatial context into which the disparate elements of an experience can be bound. Given how closely imagined experiences match episodic

\footnotetext{
$\overline{{ }^{4} \text { The patient }}$ who performed at control level had some remaining hippocampal tissue, and the authors suppose that it was this remnant that permitted imagining the future.
} 
memories, the absence of this function mediated by the hippocampus may also fundamentally affect the ability to vividly reexperience the past." They go on to state that their findings challenge the standard theory of memory consolidation but "accord well with suggestions that the hippocampus plays a critical role in imagining experiences through the provision of spatial context, in perpetuity." It is particularly important that this study controlled for such things as salience, a sense of presence, the anterograde deficit of the patients, and any concerns one might have about the veridicality of remote memory retrievals. In so doing, this method offers a powerful approach to answering questions about the role of the hippocampus in episodic memory, and we imagine that future studies using this kind of paradigm will be important in deciding between the merits of SMC and MTT.

\section{Semantic memory}

In contrast to the controversy regarding episodic memory, there is little dispute about the fate of semantic memory. Consistent with both theories, RA for semantic information, whether for facts about oneself, about public events, personalities, or even vocabulary (see Table 1 in [55]), is either spared or confined to a period of about 10 years if the damage is limited primarily to the hippocampal formation. RA can be more extensive if the damage includes other MTL and neocortical structures, reaching the same level as autobiographical memory loss in the latter case, or possibly exceeding it in the case of patients from the series of Bayley et al. [5, 6]; Bayley et al. 2006). In a cross-sectional and longitudinal study of semantic memory for famous names and vocabulary in patients with $\mathrm{AD}$, Westmacott et al. [92] showed that the extent and severity of RA increased with disease progression, suggesting that RA for semantic memory is related to the extent of neocortical atrophy.

Complicating the picture is the fact that semantic and episodic memories are not independent of one another. Semantic memory, for example, may benefit from episodic memory. Westmacott and Moscovitch [91] reported that episodic memory contributes to performance on tests of semantic memory. Thus, reading times and categorization by profession for famous names is faster and more accurate if the name is associated with a recollection that is of personal significance to the individual. For example, Elvis Presley may be associated with a personal visit to Graceland, whereas Frank Sinatra holds no such personal associations. Performance favors Elvis Presley, although both people are equally famous. In surveying the population, Westmacott and Moscovitch found that a common set of famous people can elicit such associations in over $80 \%$ of the population, whereas another set of equally famous people rarely elicits them. Importantly, the distinction between these two sets is absent or greatly diminished in amnesic people, and their performance on the two semantic tasks, naming and categorization, show no difference between the two sets of names.

In recent work, Moscovitch et al. (in preparation) found that in normal people, the extent to which recollection contributes to semantic memory for names and for public events diminishes with time, reaching an asymptote after 510 years, indicating that the facts are retained but the episodic component is absent. The temporal gradient found on tests of semantic memory in people with damage restricted to the MTL may reflect the loss of this episodic component.

\section{Studies with animals}

Because of the control over conditions during acquisition and retention, and over the location and size of lesions, studies of nonhuman mammals may help resolve some of the problems encountered in the human literature. Over the years a number of paradigms have been developed to study RA, primarily in rodents, and have yielded the same three patterns of RA after lesions to the hippocampus and related MTL structures in humans: RA with a temporal gradient, extensive RA with no gradient, and no RA. For example, several studies on contextual fear conditioning (e.g., [28]) and socially acquired taste preferences (e.g., [93]) have reported temporally graded RA in rats with hippocampal lesions. By contrast, in studies of remote spatial memory, the typical finding is temporally extensive RA either without a gradient (e.g., [58]), or with poorest memory for the most remote time periods [94]. Finally, no RA is reported on tasks in which there is no anterograde loss after hippocampal lesions, such as procedural learning, single object discrimination, or conditioning to unimodal stimuli, such as tones.

In reviewing the evidence, Rosenbaum et al. [71] accounted for much of it by positing that tests dependent on relational context (e.g., allocentric and configural spatial cues) produced a temporally extensive RA, whereas tests that are less dependent on relational context (tone, taste, or smell of conditioning stimulus) produced either a temporally graded RA or no RA. Recent studies have confirmed this hypothesis. Thus, memory for a hidden platform in a water maze (e.g., $[9,45,82]$ or for the rewarded side in a cross maze [94, 95], both open to a complex environment, was impaired after hippocampal lesions even if acquisition was months earlier. RA was not influenced by lesion size. On the other hand, memory for particular objects either showed a temporal gradient or no memory loss at all after hippocampal lesions [58], but a temporally extensive RA after perirhinal lesions in both rats [17, 57] and monkeys [84]. 
If we consider memories dependent on relational context to be analogous to episodic memory in humans, and the other two types of memory to be analogous to familiarity or semantic memory, then the general pattern of results resembles that found in humans with hippocampal lesions. This line of reasoning suggests a new interpretation of the temporal gradient observed in certain tasks such as contextual fear conditioning and acquired food preference. Rather than view the temporal gradient as evidence in favor of SMC, we can interpret it as evidence of transformation of memory representations over time from ones that are context-dependent shortly after acquisition, and rely on the hippocampus, to ones that are less dependent on context afterward, and, thus, exist independently of the hippocampus. Consistent with the latter interpretation, a number of investigators have shown that sensitivity to contextual detail diminishes with time, such that animals generalize conditioned behaviors to novel contexts as the retention interval increases. This result has been obtained both in the conditioned fear paradigm ([4, 39, 47]; Houston et al. 1999; Wiltgen and Silva, submitted for publication; Winocur and Moscovitch, submitted for publication) and in a socially acquired food preference task (Winocur and Moscovitch, submitted for publication). Similar transformations can occur with respect to complex spatial memories. With time and experience spatial memories are also transformed from contextually dependent ones to more schematic ones that can be sustained by extrahippocampal structures. In line with this interpretation, Winocur et al. [94, 95] found that rats reared in a complex environment learned the rewarded locations in that environment, and retained those memories even after hippocampal lesions. The same rats, however, were impaired in acquiring new spatial memories in a different environment. These results are consistent with reports of the effects of hippocampal lesions on remote spatial memory in humans, who can also navigate normally in an environment learned long before they sustained their damage. Although they base their navigation on a schematic, map-like representation of the environment, they do not retain details, which prevents them from recreating the original environment and reexperiencing it in rich detail. The absence of a detailed representation may also prevent rodents and humans from navigating their environment with optimum efficiency. Thus, even those rats with hippocampal lesions who navigated the complex spatial maze with relatively few errors chose less efficient routes to rewarded locations than did intact animals (Winocur and Moscovitch, in preparation).

The transformation hypothesis also helps explain some intriguing results based on the so-called reconsolidation paradigm. Once reactivated, a memory trace that was presumably consolidated and no longer dependent on the hippocampus becomes labile and susceptible to hippocam- pal lesions shortly after it has been reactivated [34]. In other words, once retrieved, a memory trace needs to be reconsolidated or consolidated anew. We would suggest that reconsolidation is a demonstration of the dynamic nature of memory, always capable of being transformed depending on the availability of cues. Reexposing the rat to the initial training environment reactivates the original, context-dependent memory representation that relies on the hippocampus, and memory for the task once again becomes susceptible to hippocampal lesions. As already noted, sensitivity to context in contextual fear conditioning is diminished with time, in keeping with its increasing independence of the hippocampus. However, providing a reminder restores that sensitivity (Winocur et al., in preparation).

\section{Hippocampal activation during retrieval of remote memory?}

Episodic memory

Most neuroimaging studies of autobiographical (episodic) memory report equivalent activation in the MTL to retrieval of recent and remote episodic memory, especially in the hippocampus, as predicted by MTT and contrary to SMC. This pattern of activation is obtained in PET and fMRI regardless of the interval, which varies from days $[69,80]$ to weeks ([37], in parahippocampal cortex) to decades [75] or the particular procedures used to elicit and test autobiographical memories: recognition of sentences describing events [40, 41, 43, 69] and reexperiencing events in response to cue words $[1,2,10,21,75]$, generic sentences [69], and family photos [18]. Both hippocampi are typically activated in comparison to a variety of baseline tasks. Even in a person with hippocampal lesions who was amnesic from early childhood, activation of residual hippocampal tissue was found for the very few remote episodic memories he had [43].

One criticism leveled against such neuroimaging studies is that hippocampal activation might be associated with the encoding of memories retrieved in the scanner, rather than with retrieval of the memories themselves. A number of controls suggest this is not the case. First, hippocampal activations are not obtained for semantic or generic memories retrieved in the scanner, which would presumably also be encoded [40, 41, 43, 69, 87]. Second, the same pattern of hippocampal activation during retrieval is found even when the baseline (or comparison) event involves generating a detailed imaginary scenario of an event that participants never experienced [18]. This finding was recently replicated in a study comparing real and imagined events ([74]; Cox et al., in preparation), even though the 
imagined events were highly detailed and contained the same components as real autobiographical experiences (e.g., including familiar people, places, and objects within the imagined event). What is most interesting is that in the study of Gilboa et al. [18], although there was equivalent activation for vivid recent and remote memories, the activations were distributed differently. Activations associated with recent memories clustered at the anterior end of the hippocampus, whereas those associated with remote memories were distributed throughout its length. These results using imagined events as the baseline condition are particularly striking in view of the recent finding, discussed earlier, that the hippocampus is probably critical to such imaginings [23]. This baseline condition must have activated the hippocampus, and the fact that remote memory retrievals activated the hippocampus to a greater extent shows that such retrievals constitute a very powerful activator of hippocampal circuits.

A few studies show a temporal gradient of activation $[16,66]$, but in these studies there was either no control for vividness, number of details or personal significance [85], or no effort was made to determine the contribution of these variables, of which the former two are known to vary inversely with the age of the memory. Eustache et al. [15] note that older memories are typically sketchier and more semantic than recent ones and Addis et al. [1] have shown that hippocampal activation is modulated by vividness, emotionality, and personal significance: memories rated high on those qualities in the scanner led to greater hippocampal activation. Although memory recency modulated hippocampal activity, its effects were reduced or eliminated when the above experiential factors were included as covariates. Conversely, robust modulation of hippocampal activation was observed for the three experiential qualities even when recency was included as a covariate (see also [21]). The only exception with regard to recency is a study by Maguire and Frith [41] who found a temporal gradient of activation in the right hippocampus in older, but not younger, adults. The cause of this anomaly is not apparent (see [18] for possible interpretation). Overall, these studies provide strong support for predictions based on MTT. Although hippocampal activation is moderated by variables that may be related to the age of the memory, it is important to note that no studies to date have shown a complete lack of hippocampal activation for very remote events, as SMC would predict.

\section{Semantic memory}

The neuroimaging evidence on semantic memory is less consistent than that on episodic memory. There have been fewer studies examining remote memory for personal semantics or public knowledge of people and events, and those have found either no hippocampal activation; hippocampal activation without a gradient for personal semantics, knowledge of public events, [40] and famous faces [7, 27, 35]; or a temporally graded activation in right entorhinal cortex to famous faces [22] and in right parahippocampal cortex to names [12]. In all cases, the time range sampled was greater than 20 years, extending as high as 50 years [22]. The source of the discrepancy is difficult to determine at present (see [55] for discussion).

The temporal gradient observed in some studies of semantic memory is consistent with MTT and SMC in that they both suggest that hippocampal involvement in retention and retrieval of semantic memory diminishes with time. Other studies, reporting the absence of a temporal gradient favor MTT to the extent that such gradients reflect the contribution of an episodic component.

In addition to these studies of semantic memory, there are a handful of functional neuroimaging studies on remote spatial memory. Maguire et al. [42] tested the ability of experienced London taxi drivers to find new routes from one location to another when familiar routes were blocked. They report hippocampal activation associated with success in novel wayfinding, but the region of activation is in the parahippocampal cortex, not in the hippocampus itself. Likewise, in a test that required participants to reexperience an event in a particular location (combined spatial and autobiographical memory test), Niki and Luo [63] found greater activation in the left parahippocampal gyrus when contrasting recent (within 2 years) detailed events with remote ( 7 years) detailed events. In a complex study contrasting different types of spatial, semantic, and episodic memory acquired recently (within weeks at most) or 4 years earlier, Mayes et al. [46] report activation in the right body and head of the hippocampus when contrasting the reliving of an episode in a particular place (static episode) with recalling the location of six towns on a map (semantic spatial), with no effect of age. The hippocampal activation might be related to the spatial nature of the memory, or to the vividness (number of details of the environment and autobiographical context) of the experience, much as was found for hippocampal activation of episodic memory (see above). In both of the studies, however, there was also activation in precuneus, parietal, parahippocampal, and posterior cingulate cortex, structures that appear to be part of a spatial network [72].

Medial temporal regions may be particularly involved in recollection of the spatial component of a memory, although the particular site of activation may differ depending upon the specific requirements of the task. Hayes et al. [24] had subjects view a videotaped tour of four houses, and then compared activation for three aspects of episodic memory; memory for objects, temporal order, and spatial location of objects. Whereas activation in the hippocampus 
proper was similar across the three conditions, bilateral parahippocampal cortex activation was greater for spatial location compared to object and temporal order information. Several recent studies in our laboratory (Ryan et al., in preparation; Hoscheidt et al., in preparation) suggest that this difference is observed regardless of whether the source of the to-be-recalled information is episodic or semantic; tasks that emphasize spatial location or spatial relations show greater activation in parahippocampal cortex, hippocampus proper, or both. However, the activation within the hippocampus proper during semantic retrieval occurs in the anterior third of the hippocampus, whereas activation associated with episodic retrieval is clearly posterior, often extending into adjacent parahippocampal cortex.

Rosenbaum et al. [72] used a version of the Toronto Public Places Test, modified for scanning. They found that the hippocampus proper was not activated on any of the tests more than on the baseline control task, although the parahippocampal cortex was active, as noted by Maguire et al. The level of activation in extrahippocampal regions varied with the particular demands of each task. For example, the superior-medial parietal cortex was implicated more in egocentric tests of spatial memory, such as landmark sequencing, whereas the retrosplenial cortex was implicated more on allocentric tests, such as vector mapping, distance judgments, and proximity judgments. Memory for familiar places activates some of the same regions and also parts of anterior, temporal cortex [20]. The only evidence of hippocampal activation to familiar places comes from studies in which there is some personal, spatial reasoning [19, 33, 63], suggesting that the experiential component might be crucial.

Neuroimaging evidence on remote spatial memory is consistent with both MTT and SMC, which claim, for different reasons, that remote spatial memories can exist independently of the hippocampus. As we suggested elsewhere [53-55, 70, 94, 95], it is possible that remote spatial memories existing independently of the hippocampus are coarser than those dependent on the hippocampus.

\section{Conclusion}

The literature provides relatively clear answers to the questions we raised at the outset to distinguish the SMC and MTT:

1. Are the remote memories retrieved by people with MTL amnesia as fully detailed as the remote memories retrieved by intact individuals?

When the most sensitive methods are used to assess remembered detail, the data show that remote memories retrieved by amnesic patients are detail-poor compared to the remote memories retrieved by appropriate control subjects.
In both the spatial and nonspatial domains, remote memories retrieved by amnesic patients lack rich contextual detail.

2. Is the hippocampal complex activated by retrieval of remote episode memories?

The hippocampal complex is most definitely activated during the retrieval of remote episodic memory. This does not appear to reflect mere reencoding that might occur when an old memory is brought back to conscious awareness.

3. Do episodic and semantic memories suffer the same fate during consolidation and in amnesia?

Most definitely not. In accordance with MTT, but not with SMC, episodic and semantic memories fare quite differently over the course of consolidation.

We submit that the empirical data are not consistent with SMC. They are, however, consistent with many of the assertions of MTT. However, there are aspects of MTT, at least as first formulated, that are not so well supported. We initially argued that the extent of the gradient in RA should vary as a function of the size of the hippocampal complex lesion [61]. This prediction was related to the notion that each time an episodic memory is retrieved a "replicate" trace is created within the hippocampal complex, spreading out the representation of that memory. This spread would mean that larger lesions are required to knock out older memories, on average.

However, the evidence in support of these particular predictions is inconsistent ([32], but see Gilboa et al. 2005), suggesting that the specific mechanism we proposed to account for the continuing hippocampal involvement in episodic memory over time might have been incorrect. The evidence strongly supports this continuing involvement, however, so we should consider other mechanisms that would accomplish this function.

Trace replication is but a specific example of the more general idea that when an episode memory is reinstated, its representation within hippocampal circuits is altered. Other forms of alteration are possible, such as strengthening the existing trace, or changing it by incorporating some new information as represented in additional neuronal circuits. In most circumstances this alteration will lead both to an incorporation of new information into the memory trace, and a concomitant strengthening of the trace as a result of the reconsolidation process itself. Further discussion of this possibility is beyond the scope of the present review, but we imagine that development of this idea and the linkages between MTT and reconsolidation will be well worth pursuing in the future.

The evidence reviewed in this paper provides a unified framework for conceptualizing hippocampal-neocortical interactions [53, 54, 71]. In this framework, detailed 
representations of remote events (episodic, autobiographical memory in humans and context-dependent memory in animals), including rich spatial representations of environments, are hippocampally dependent, whereas semantic memories (context-free memories) and schematic or coarse representations of the topography (sufficient to support navigation) can exist independently of the hippocampus.

In many ways, the theoretical position espoused for the role of the hippocampus in remote memory is congruent with the emerging view of the hippocampus in recent (anterograde) memory in humans and other animals: It is needed to represent information that supports recollection of the past, but not context-independent familiarity with it ([13, 14, 26, 52, 96], but see Wais et al. 2006). Insofar as memories reflect detailed information, they will continue to be dependent on the hippocampus. Memories, however, are typically transformed with time, losing details and becoming more schematic and scripted. What is more, retrieval appears to reinstantiate a previously stored and consolidated memory, which can then be transformed by the subsequent retrieval context.

Acknowledgment Preparation of this paper was supported by grants from National Institute of Neurological Disorders and Stroke (NS044107) and the Arizona Alzheimer's Research Center (Department of Health Services, State of Arizona, HB2354) to L.R. and L.N., and a Canadian Institute of Health Research grant to M.M. and G.W.

\section{References}

1. Addis DR, Moscovitch M, Crawley AP, McAndrews MP (2004) Recollective qualities modulate hippocampal activation during autobiographical memory retrieval. Hippocampus 14:752-762

2. Addis DR, McIntosh AR, Moscovitch M, Crawley AP, McAndrews MP (2004) Characterizing the spatial and temporal features of autobiographical memory retrieval networks: a partial least squares approach. Neuroimage 23:1460-1471

3. Alvarez P, Squire LR (1994) Memory consolidation and the medial temporal lobe: a simple network model. Proc Natl Acad Sci U S A 91:7041-7045

4. Balogh SA, Radcliffe RA, Logue SF, Wehner JM (2002) Contextual and cued fear conditioning in C57BL/6J and DBA/2J mice: context discrimination and the effects of retention interval. Behav Neurosci 116:947-957

5. Bayley PJ, Hopkins RO, Squire LR (2003) Successful recollection of remote autobiographical memories by amnesic patients with medial temporal lobe lesions. Neuron 38:135-144

6. Bayley PJ, Gold JJ, Hopkins RO, Squire LR (2005) The neuroanatomy of remote memory. Neuron 46:799-810

7. Bernard FA, Bullmore ET, Graham KS, Thompson SA, Hodges JR, Fletcher PC (2004) The hippocampal region is involved in successful recognition of both remote and recent famous faces. Neuroimage 22:1704-1714

8. Cipolotti L, Shallice T, Chan D, Fox N, Scahill R, Harrison G, Stevens J, Rudge P (2001) Long-term retrograde amnesia: the crucial role of the hippocampus. Neuropsychologia 39:151-172

9. Clark RE, Broadbent NJ, Squire LR (2005) Impaired remote spatial memory after hippocampal lesions despite extensive training beginning early in life. Hippocampus 15:340-346

10. Conway MA, Turk DJ, Miller SL, Logan J, Nebes RD, Meltzer CC, Becker JT (1999) A positron emission tomography (PET) study of autobiographical memory retrieval. Memory 5-6:679-702

11. Corkin S (2005) What's new with the amnesic patient H.M.? Nat Rev Neurosci 3:153-160

12. Douville K, Woodard JL, Seidenberg M, Miller SK, Leveroni CL, Nielson KA, Franczak M, Antuono P, Rao SM (2005) Medial temporal lobe activity for recognition of recent and remote famous names: an event-related fMRI study. Neuropsychologia 43:693703

13. Eichenbaum H (2004) Hippocampus: cognitive processes and neural representations that underlie declarative memory. Neuron 44:109-120

14. Eldridge LL, Knowlton BJ, Furmanski CS, Bookheimer SY, Engel SA (2000) Remembering episodes: a selective role for the hippocampus during retrieval. Nat Neurosci 3:1149-1152

15. Eustache F, Piolino P, Giffard B, Viader F, De La Sayette V, Baron JC, Desgranges B (2003) In the course of time: a PET study of the cerebral substrates of autobiographical amnesia in Alzheimer's disease. Brain 127:1549-1560

16. Fink GR, Markowitsch HJ, Reinkemeier M, Bruckbauer $T$, Kessler J, Heiss WD (1996) Cerebral representation of one's own past: neural networks involved in autobiographical memory. J Neurosci 16:4275-4282

17. Gaskin S, Tremblay A, Mumby DG (2003) Retrograde and anterograde object recognition in rats with hippocampal lesions. Hippocampus 13:962-969

18. Gilboa A, Winocur G, Grady CL, Hevenor SJ, Moscovitch M (2004) Remembering our past: functional neuroanatomy of recollection of recent and very remote personal events. Cereb Cortex 14:1214-1225

19. Goel V, Makale M, Grafman J (2004) The hippocampal system mediates logical reasoning about familiar spatial environments. J Cogn Neurosci 16:654-664

20. Gorno-Tempini ML, Price CJ (2001) Identification of famous faces and buildings: a functional neuroimaging study of semantically unique items. Brain 124:2087-2097

21. Graham KS, Lee ACH, Brett M, Patterson K (2003) The neural basis of autobiographical and semantic memory: new evidence from three PET studies. Cogn Affect Behav Neurosci 3:234254

22. Haist F, Gore JB, Mao H (2001) Consolidation of human memory over decades revealed by functional magnetic resonance imaging. Nat Neurosci 4:1139-1145

23. Hassabis D, Kumaran D, Vann SD, Maguire EA (2007) Patients with hippocampal amnesia can't imagine new experiences. Proc Natl Acad Sci USA 104:1726-1731

24. Hayes SM, Ryan L, Schnyer D, Nadel L (2004) An fMRI study of episodic memory: retrieval of object, spatial, and temporal information. Behav Neurosci 118:885-896

25. Hodges JR, Graham KS (2001) Episodic memory: insights from semantic dementia. Philos Trans R Soc Lond B 356:1423-1434

26. Holdstock JS, Mayes AR, Roberts N, Cezayirli E, Isaac CL, O'Reilly RC, Norman KA (2002) Under what conditions is recognition spared relative to recall after selective hippocampal damage? Hippocampus 12:341-351

27. Kapur N, Friston KJ, Young A, Frith CD, Frackowiak RS (1995) Activation of human hippocampal formation during memory for faces: a PET study. Cortex 31:99-108

28. Kim JJ, Fanselow MS (1992) Modality-specific retrograde amnesia of fear. Science 256:675-677

29. Kopelman MD (2002) Retrograde amnesia. In: Baddeley AD, 
Kopelman MD, Wilson BA (eds) Handbook of memory disorders, 2nd edn. Wiley, London, UK, pp 189-208

30. Kopelman MD, Kapur N (2001) The loss of episodic memories in retrograde amnesia: single-case and group studies. Philos Trans R Soc Lond B 356:1409-1421

31. Kopelman MD, Lasserson D, Kingsley DR, Bello F, Rush C, Stanhope N, Stevens TG, Goodman G, Buckman JR, Heilpern G, Kendall BE, Colchester ACF (2003) Retrograde amnesia and the volume of critical brain structures. Hippocampus 13:879-891

32. Kopelman MD, Wilson BA, Baddeley AD (1989) The Autobiographical Memory Interview: a new assessment of autobiographical and personal semantic memory in amnesic patients. J Clin Exp Neuropsychol 5:724-744

33. Kumaran D, Maguire EA (2005) The human hippocampus: cognitive maps or relational memory? J Neurosci 25:7254-7259

34. Land C, Bunsey M, Riccio DC (2000) Anomalous properties of hippocampal lesion-indiced retrograde amnesia. Psychobiology 28:476-485

35. Leveroni CL, Seidenberg M, Mayer AR, Mead LA, Binder JR, Rao SM (2000) Neural systems underlying the recognition of familiar and newly learned faces. J Neurosci 20:878-886

36. Levine B, Svoboda E, Hay JF, Winocur G, Moscovitch M (2002) Aging and autobiographical memory: dissociating episodic from semantic retrieval. Psychol Aging 17:677-689

37. Levine B, Turner GR, Tisserand D, Hevenor SJ, Graham SJ, McIntosh AR (2004) The functional neuroanatomy of episodic and semantic autobiographical remembering: a prospective functional MRI study. J Cogn Neurosci 16:1633-1646

38. Lewis DJ (1979) Psychobiology of active and inactive memory. Psychol Bull 86:1054-1083

39. MacArdy EA, Riccio DC (1995) Time-dependent changes in the effectiveness of a non-contingent footshock reminder. Learn Motiv 26:29-42

40. Maguire EA (2001) Neuroimaging studies of autobiographical event memory. Philos Trans R Soc Lond B 356:1441-1451

41. Maguire EA, Frackowiak RSJ, Frith CD (1997) Recalling routes around London: activation of the right hippocampus in taxi drivers. J Neurosci 7:7103-7110

42. Maguire EA, Frith CD (2003) Lateral asymmetry in the hippocampal response to the remoteness of autobiographical memories. J Neurosci 23:5302-5307

43. Maguire EA, Vargha-Khadem F, Mishkin M (2001) The effects of bilateral hippocampal damage on fMRI regional activations and interactions during memory retrieval. Brain 124:1156-1170

44. Marr D (1971) Simple memory: a theory for archicortex. Philos Trans R Soc Lond B 262:23-81

45. Martin SJ, de Hoz L, Morris RGM (2005) Retrograde amnesia: neither partial nor complete hippocampal lesions in rats result in preferential sparing of remote spatial memory, even after reminding. Neuropsychologia 43:609-624

46. Mayes AR, Montaldi D, Spencer TJ, Roberts N (2004) Recalling spatial information as a component of recently and remotely acquired episodic or semantic memories: an fMRI study. Neuropsychology 18:426-441

47. McAllister WR, McAllister DE (2006) Recovery of conditioned fear by a single postextinction shock: effect of similarity of shock contexts and of time following extinction. Learn Behav 34:44-49

48. McClelland JL, McNaughton BL, O'Reilly RC (1995) Why there are complementary learning systems in the hippocampus ands neocortex: insights from the successes and failures of connectionist models of learning and memory. Psychol Rev 102:419-436

49. Moscovitch M (1992) Memory and working with memory: a component process model based on modules and central systems. J Cogn Neurosci 4:257-267
50. Moscovitch M (1995) Recovered consciousness: a hypothesis concerning modularity and episodic memory. J Clin Exp Neuropsychol 17:276-291

51. Moscovitch DA, McAndrews MP (2002) Material-specific deficits in 'remembering' in patients with unilateral temporal lobe epilepsy and excisions. Neuropsychologia 40:1335-1342

52. Moscovitch M, Rosenbaum RS, Gilboa A, Addis DR, Westmacott R, Grady CL, McAndrews MP, Winocur G, Nadel L (2005) Functional neuroanatomy of remote episodic, semantic and spatial memory: a unified account based on multiple trace theory. J Anat 207:35-66

53. Moscovitch M, Westmacott R, Gilboa A, Addis D, Rosenbaum RS, Viskontas I, Priselac S, Svoboda E, Ziegler M, Black S, Gao F, Grady C, Freedman M, Köhler S, Leach L, Levine B, McAndrews MP, Nadel L, Proulx G, Richards B, Ryan L, Stokes K, Winocur W (2005) Hippocampal complex contribution to retention and retrieval of recent and remote episodic and semantic memories: evidence from behavioral and neuroimaging studies of healthy and brain-damaged people. In: Ohta N, MacLeod CM, Uttl B (eds) Dynamic cognitive processes. Springer-Verlag, Tokyo, pp 333-380

54. Moscovitch M, Nadel L (1998) Consolidation and the hippocampal complex revisited: in defense of the multiple-trace model. Curr Opin Neurobiol 8:297-300

55. Moscovitch M, Nadel L, Winocur G, Gilboa A, Rosenbaum S (2006) The cognitive neuroscience of remote episodic, semantic and spatial memory. Curr Opin Neurobiol 16:179-190

56. Moss HE, Kopelman MD, Cappelletti M, de Mornay Davies P, Jaldow E (2003) Lost for words or loss of memories? Autobiographical memory in semantic dementia. Cogn Neuropsychol 20:703-732

57. Mumby DG, Astur RS, Weisend MP, Sutherland RJ (1999) Retrograde amnesia and selective damage to the hippocampal formation: memory for places and object discriminations. Behav Brain Res 106:97-107

58. Mumby DG, Glenn MJ (2000) Anterograde and retrograde memory for object discriminations and places in rats with perirhinal cortex lesions. Behav Brain Res 114:119-134

59. Murre JMJ, Graham KS, Hodges JR (2001) Semantic dementia: relevance to connectionist models of long-term memory. Brain 124:647-675

60. Nadel L (2007) Multiple memory systems: a new view. In: Byrne $\mathrm{J}$ (ed) Learning and memory: a comprehensive reference. Elsevier, Amsterdam (in press)

61. Nadel L, Moscovitch M (1997) Consolidation, retrograde amnesia and the hippocampal formation. Curr Opin Neurobiol 7:217-227

62. Nadel L, Moscovitch M (1998) Hippocampal contribution to cortical plasticity. Neuropharmacology 37:431-440

63. Niki K, Luo J (2002) An fMRI study on the time-limited role of the medial temporal lobe in long-term topographical autobiographic memory. J Cogn Neurosci 14:500-507

64. O'Keefe J, Nadel L (1978) The hippocampus as a cognitive map. Clarendon, Oxford

65. Penfield W, Milner B (1958) Memory deficit produced by bilateral lesions in the hippocampal zone. A M A Arch Neurol Psych 79:475-497

66. Piefke M, Weiss PH, Zilles K, Markowitsch HJ, Fink GR (2003) Differential remoteness and emotional tone modulate the neural correlates of autobiographical memory. Brain 126:650-668

67. Poreh A, Winocur G, Moscovitch M, Backon M, Goshen E, Ram Z, Feldman Z (2006) Anterograde and retrograde amnesia in a person with bilateral fornix lesions following removal of a colloid cyst. Neuropsychologia 44:2241-2248

68. Reed JM, Squire LR (1998) Retrograde amnesia for facts and events: findings from four new cases. J Neurosci 18:3943-3954 
69. Rekkas PV, Constable RT (2005) Evidence that autobiographic memory retrieval does not become independent of the hippocampus: an fMRI study contrasting very recent with remote events. J Cogn Neurosci 17:1950-1961

70. Rosenbaum RS, Priselac S, Kohler S, Black SE, Gao F, Nadel L, Moscovitch M (2000) Remote spatial memory in an amnesic person with extensive bilateral hippocampal lesions. Nat Neurosci 3:1044-1048

71. Rosenbaum RS, Winocur G, Moscovitch M (2001) New views on old memories: re-evaluating the role of the hippocampal complex. Behav Brain Res 127:183-197

72. Rosenbaum RS, Ziegler M, Winocur G, Grady CL, Moscovitch M (2004) I have often walked down this street before: fMRI studies on the hippocampus and other structures during mental navigation of an old environment. Hippocampus 14:826-835

73. Russell WR, Nathan PW (1946) Traumatic amnesia. Brain 69:280-300

74. Ryan L, Cox C, Patterson D, Nadel L (2005) Comparing fMRI activation of real and imagined autobiographical event memories. Organization for Human Brain Mapping, Toronto (June)

75. Ryan L, Nadel L, Keil K, Putnam K, Schnyer D, Trouard T, Moscovitch M (2001) Hippocampal complex and retrieval of recent and very remote autobiographical memories: evidence from functional magnetic resonance imaging in neurologically intact people. Hippocampus 11:707-714

76. Schacter DL, Addis DR (2007) Constructive memory: the ghosts of past and future. Nature 445:27

77. Scoville WB, Milner B (1957) Loss of recent memory after bilateral hippocampal lesion. J Neurol Neurosurg Psychiatry 20:11-21

78. Squire LR, Alvarez P (1995) Retrograde amnesia and memory consolidation: a neurobiological perspective. Curr Opin Neurobiol 5:169-177

79. Squire LR, Cohen NJ, Nadel L (1984) The medial temporal region and memory consolidation: a new hypothesis. In: Weingartner $\mathrm{H}$, Parker E (eds) Memory consolidation. Lawrence Erlbaum, Hillsdale, NJ, pp 185-210

80. Stark CEL, Squire LR (2000) fMRI activity in the medial temporal lobe during recognition memory as a function of study-test interval. Hippocampus 10:329-337

81. Steinvorth S, Levine B, Corkin S (2005) Medial temporal lobe structures are needed to re-experience remote autobiographical memories: evidence from H.M. \& W.R. Neuropsychologia 43:479-496

82. Sutherland RJ, Weisend MP, Mumby D, Astur RS, Hanlon F, Koerner A, Thomas MJ, Wu Y, Moses SN, Cole C, Hamilton
DA, Hoesing JM (2001) Retrograde amnesia after hippocampal damage: recent vs. remote memories in two tasks. Hippocampus $11: 27-42$

83. Teyler TJ, DiScenna P (1985) The role of the hippocampus in memory: a hypothesis. Neurosci Biobehav Rev 9:377-389

84. Thornton JA, Rothblat LA, Murray EA (1997) Rhinal cortex removal produces amnesia for preoperatively learned discrimination problems but fails to disrupt postoperative acquisition and retention in rhesus monkeys. J Neurosci 17:8536-8549

85. Tsukiura T, Fujii T, Okuda J, Ohtake H, Kawashima R, Itoh M, Fukuda H, Yamadori A (2002) Time-dependent contribution of the hippocampal complex when remembering the past: a PET study. NeuroReport 13:2319-2323

86. Tulving E (2002) Episodic memory: from mind to brain. Annu Rev Psychol 53:1-25

87. Viard A, Piolino P, Desgranges B, Chételat G, Lebreton B, Young A, De La Sayette V, Eustache F (2007) Hippocampal activation for autobiographical memories over the entire lifetime in healthy aged subjects: an fMRI study. Cereb Cortex (in press)

88. Warrington EK, Sanders HI (1971) The fate of old memories. Q J Exp Psychol 23:432-442

89. Warrington EK, Weiskrantz L (1968) New method of testing longterm retention with special reference to amnesic patients. Nature 217:972-974

90. Warrington EK, Weiskrantz L (1970) Amnesic syndrome: consolidation or retrieval? Nature 228:628-630

91. Westmacott R, Moscovitch M (2003) The contribution of autobiographical significance to semantic memory. Mem Cogn 31:761-774

92. Westmacott R, Black SE, Freedman M, Moscovitch M (2004) The contribution of autobiographical significance to semantic memory: evidence from Alzheimer's disease, semantic dementia, and amnesia. Neuropsychologia 42:25-48

93. Winocur G (1990) Anterograde and retrograde amnesia in rats with dorsal hippocampal or dosomedial thalamic lesions. Behav Brain Res 38:145-154

94. Winocur G, Moscovitch M, Caruana DA, Binns MA (2005) Retrograde amnesia in rats with lesions to the hippocampus on a test of spatial memory. Neuropsychologia 43:1580-1590

95. Winocur G, Moscovitch M, Fogel S, Rosenbaum RS, Sekeres M (2005) Preserved spatial memory after hippocampal lesions: effects of extensive experience in a complex environment. Nat Neurosci 8:273-275

96. Yonelinas AP (2002) The nature of recollection and familiarity: a review of 30 years of research. J Mem Lang 46:441-517 